\title{
An Experimental Introduction to Basic Principles of the Interaction of Electromagnetic Radiation with Matter
}

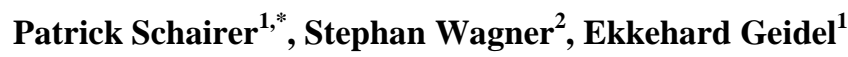 \\ ${ }^{1}$ Didactics of Chemistry, Julius-Maximilians-University Würzburg, Würzburg, Germany \\ ${ }^{2}$ Institute of Inorganic Chemistry, Julius-Maximilians-University Würzburg, Würzburg, Germany \\ *Corresponding author: patrick.schairer@uni-wuerzburg.de
}

\begin{abstract}
To understand basic principles about the interaction of electromagnetic radiation with matter is often a challenge in chemical education due to the difficult theoretical background of this topic. The present contribution therefore offers an experimental based introduction into the basic principles of UV/Vis spectroscopy following a three-step strategy. The starting point is to construct a simple self-built spectrometer working within the visible range of light. Learners can explore the most important components of such a device and understand their functions without previous knowledge. In a second step, emission spectra of different common light sources are investigated and compared. Finally, spectroscopic experiments are suggested for chemical education such as the qualitative detection of cations and the quantitative analysis of the dye carmine in food. This context-based introduction links chemical applications with the everyday life. It can be presumed that this way, learners are provided an easier access to radiation-matter interaction.
\end{abstract}

Keywords: UV/Vis spectroscopy, low-cost spectrometer, flame test, quantitative analysis, carmine

Cite This Article: Patrick Schairer, Stephan Wagner, and Ekkehard Geidel, "An Experimental Introduction to Basic Principles of the Interaction of Electromagnetic Radiation with Matter." World Journal of Chemical Education, vol. 6, no. 1 (2018): 29-35. doi: 10.12691/wjce-6-1-6.

\section{Introduction}

Spectroscopic techniques have been proven to be one of the most powerful tools to solve problems encountered in qualitative and quantitative analytical chemistry. In the field of education, the introduction to this exciting topic of modern experimental chemistry is a highly demanding challenge. Two different strategies may be successful.

If the previous knowledge of learners about the structure of matter is more advanced, one may follow the traditional way of physical chemistry by starting from the point of view of matter. In this case, the key to understanding the fundamentals of spectroscopy is the quantization of the energy levels of matter. For example, in a molecular spectroscopy experiment the sample is placed in an electromagnetic field (e.g., light) and the sample molecules specifically absorb the energy from the radiation. As a result, these molecules are excited from a lower energy ground state $E^{\prime}$ to a higher energy excited state E". The absorbed energy corresponds to the energy difference $\Delta \mathbf{E}$ of the occurring transition:

$$
\Delta \mathbf{E}=\mathbf{E}^{\prime \prime}-\mathbf{E}^{\prime}
$$

Thus the molecule specifically absorbs radiation with a wavelength corresponding to $\Delta \mathbf{E}$. The transfer of energy from the field to the molecule occurs if Bohr's frequency condition $\Delta \mathbf{E}=\mathbf{h} \cdot v=\mathbf{h} \cdot \mathbf{c} / \lambda$ is satisfied, where $\mathrm{h}$ being Planck's constant, c the velocity of light, and $v$ and $\lambda$ the frequency and wavelength of the light. If the molecule reverts from $E^{\prime \prime}$ to $E^{\prime}$, radiation of the same frequency is emitted. Absorption and emission of electromagnetic radiation are key characteristics of matter and can be utilized in different frequency ranges in a number of powerful spectroscopic techniques.

An alternative strategy - if the pre-existing knowledge of the learners is less advanced - is to introduce the electromagnetic spectrum and the origin of transitions phenomenologically via the main components common to most dispersive spectrometers and their functionality. In this contribution we pursue the second strategy. In a first step, learners design a simple spectrometer to explore the function and operation of the main parts. In a second step, typical applications for context-based experiments utilizing UV/Vis spectroscopy are examined. Context-based learning is a key for a modern chemistry class. There are projects such as Salters Chemistry Course (U.K.) or Chemie im Kontext (Germany) which offer a context-based access to chemistry [1]. This form of learning raises the motivation of the learners and promotes their interests in chemical or interdisciplinary scientific problems [1,2,3]. Therefore, to achieve relevance of the content, examples with chemistry-related topics were chosen that are of inherent interest for learners and therefore may motivate them to learn more about spectroscopic techniques.

\section{A Self-Made, Low-Cost Spectrometer for Educational Usage}

\subsection{Concept}

The purpose of the practical self-construction of a 
spectrometer is to explore the function of each component by the learners with the help of experiments, following the basic principles of constructionism. Constructionist teaching is based on the belief that learning occurs whenever learners are actively involved in a process of knowledge construction [4].

Many suggestions and instructions for low-cost and do-it-yourself spectrometers have been made in the literature. Some of them have specific advantages, whether it is the compact and 3D-printable structure [5] or the direct graphical output of a spectrum [6]. However, the different parts of a spectrometer are often fixed or the path of light is no longer obvious once the spectrometer is setup.

Our approach is to develop a spectrometer with movable but robust parts so that learners can assemble the spectrometer within a few minutes. Necessary parts include a light source, a slit, a sample department, a diffraction grating and a detector. Additionally, it should be possible to use the single parts for specific experiments also, in order to explore their function in such an instrument. With this knowledge learners can assemble the spectrometer correctly and conduct some first, simple measurements in order to get used to the method.

\subsection{Setup}

The low-cost spectrometer has the same essential parts as a professional laboratory instrument, as is shown in Figure 1.

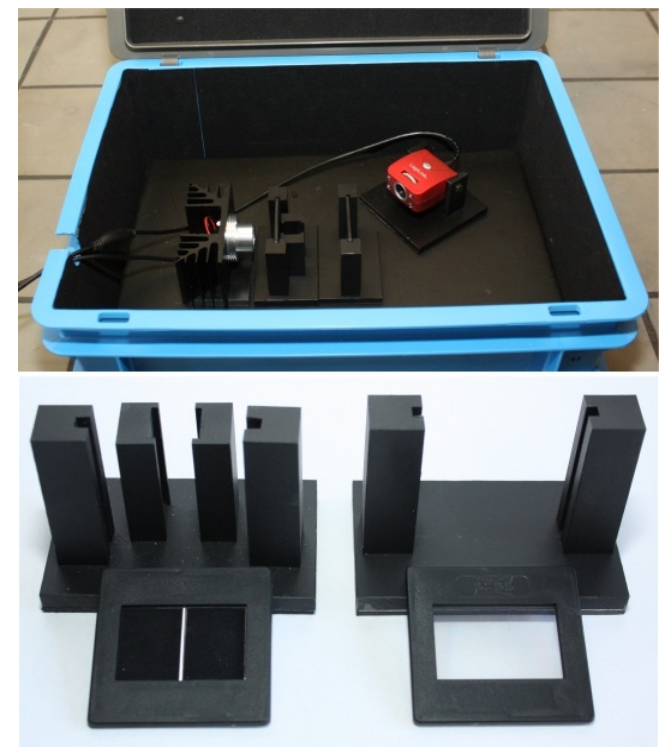

Figure 1. Spectrometer setup (top) with the essential slit, sample department and diffraction grating parts (bottom)

The light sources are common LEDs, which have a special sheathing so that they can easily be plugged into the suitable socket. All diodes are dimmable by a dimmer switch, which is connected to the socket. For absorption measurements, a white light LED is used. To calibrate the instrument there are several LEDs, which emit light of two or three specific wavelengths.

The slit is made of two razor blades which were fitted into a small frame that was originally used in an old slide projector. Black felt was used to minimize the reflection by the blades. Directly behind the frame containing the slit a cuvette holder for standard $1 \mathrm{~cm}$ cuvettes is placed. All holders are made of robust hard plastic, which is ideal for practical laboratory work.

The grating is a transmissive diffraction grit with 1000 lines per mm. Like the slit, this was also fitted into a frame, which can be placed in a holder. The grating is convertible and can also be replaced by a prism.

A common webcam (LogiLink, Germany) is used as a detector. It is connected to a personal computer installed with the free software Theremino Spectrometer developed by PhysicsOpenLab.

All of the parts are attached to holders, which have a broad and magnetic base to secure a firm but still movable positioning. The entire setup is stored in a box, as shown in Figure 1. It is lined with black felt to guarantee a dark beam path. The bottom of the box contains an iron plate, where the holders of the spectroscopic parts can be fixed.

\subsection{Studying the Functionality of Spectral Components}

Experiences have shown that many learners know how to operate a given device to get proper measurements, although they have only little knowledge about the function of the device. To avoid that learners considering laboratory instruments simply as "black boxes", a specially developed introduction course introduces them, first, to the functionality of a spectrometer and its main components and secondly to typical applications of $\mathrm{UV} / \mathrm{V}$ is spectroscopy. Regarding the knowledge of the learners, the introduction could be held within three to five days. At the beginning, the main parts of the spectrometer and their roles are analyzed. Therefore, several experiments were developed and an appropriate manual leads through the course. All experiments can be downloaded from [7].

One model experiment, showing the coherence between the width of slit and the resolution is described as an example. Slits are important to assess the performance of the monochromator and thereby the quality of the spectrum. On the one hand, the width of the entrance slit should be chosen to be as narrow as possible to ensure good resolution. On the other hand, the slit should be as wide as necessary to provide a sufficient intensity of light [8].

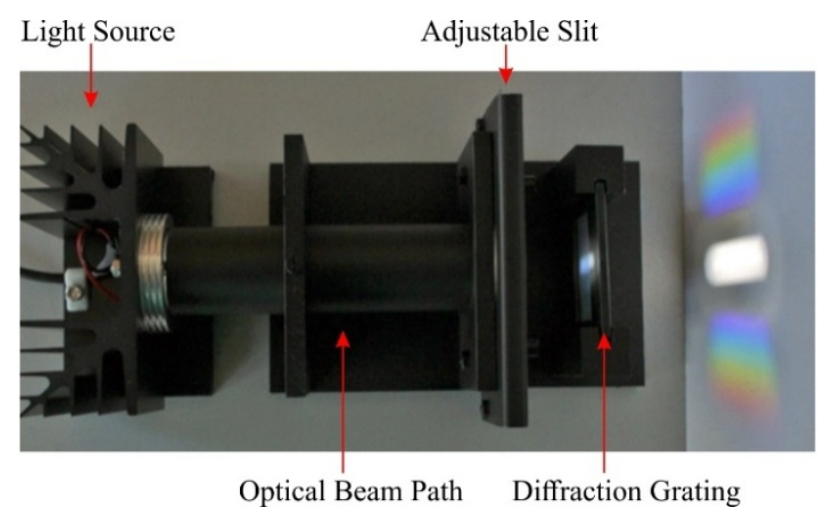

Figure 2. Setup for the slit experiment

Learners should first construct a hypothesis about how the slit's width influences spectral resolution. They can setup the slit experiment as shown in Figure 2. Here, a special optical beam path with an adjustable slit is used. 
The learners test their hypothesis by varying the width of the slit and watching the first order maximum of the diffracted light. After the experiment they draw the correct conclusion about the function of the slit and the influence of its width. The optimal width of the slit depends on the individual experimental task.

\section{Exploring the Applicability of Spectrometers by Recording Emission Spectra}

For a sample to be in thermal and radiative equilibrium with the environment at the same temperature, it is necessary for the amount of radiation absorbed from the environment to be equal to the amount emitted at all wavelengths ("blackbody" radiation). However, if the probe is at higher energy levels - as it is utilized in everyday light sources characteristic emission spectra can be recorded.

After learners have obtained knowledge about the spectrometer's function, they explore context-based applications of UV/Vis spectroscopy. In order to do so, the recording and interpretation of emission spectra of everyday light sources provide an appropriate access. For reasons of simplicity, experiments about emission spectra of single-colored LEDs are conducted in a first step.

\subsection{Emission Spectra of Various Types of LEDs}

Emission spectra of various types of light-emitting diodes (LEDs) recorded with the self-built spectrometer are shown in Figure 3. As can be seen, each LED emits light of a characteristic wavelength range and therefore with a characteristic color. In the simplest case a LED consists of a chip of semiconducting material doped with impurities to create a $\mathrm{p}-\mathrm{n}$ junction. If current flows, electrons meet holes at the junction and they fall (recombine) into a lower energy level by releasing energy as radiation (electroluminescence). The individual wavelength of the emitted light depends on the band gap energy of the semiconductor materials forming the $p-n$ junction.
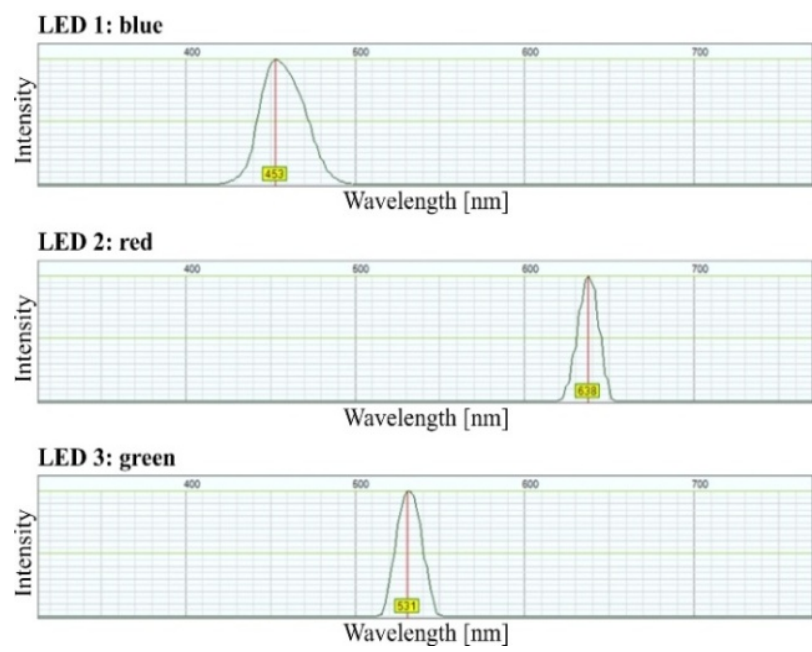

Figure 3. Emission spectra of three different types of LEDs with specific emission maxima, recorded with the self-built spectrometer

\subsection{Emission Spectra of Headlights}

In a second step, emission spectra of headlights were recorded. Many innovations concerning headlights of cars (e.g. LED, halogen, xenon) have been made in the last few years. The combination of this fact with emission spectroscopy provides a promising didactic access point. In contrast to single-colored LEDs, the different types of car headlights all appear white. Learners begin to understand that there are several types of white light by analyzing the different emission spectra of various headlights. Due to practical reasons, the emission spectra of headlights, shown in Figure 4, were recorded with a commercially available OceanOptics USB-650-VIS-NIR Red Tide spectrometer equipped with an optical fiber patch cord.
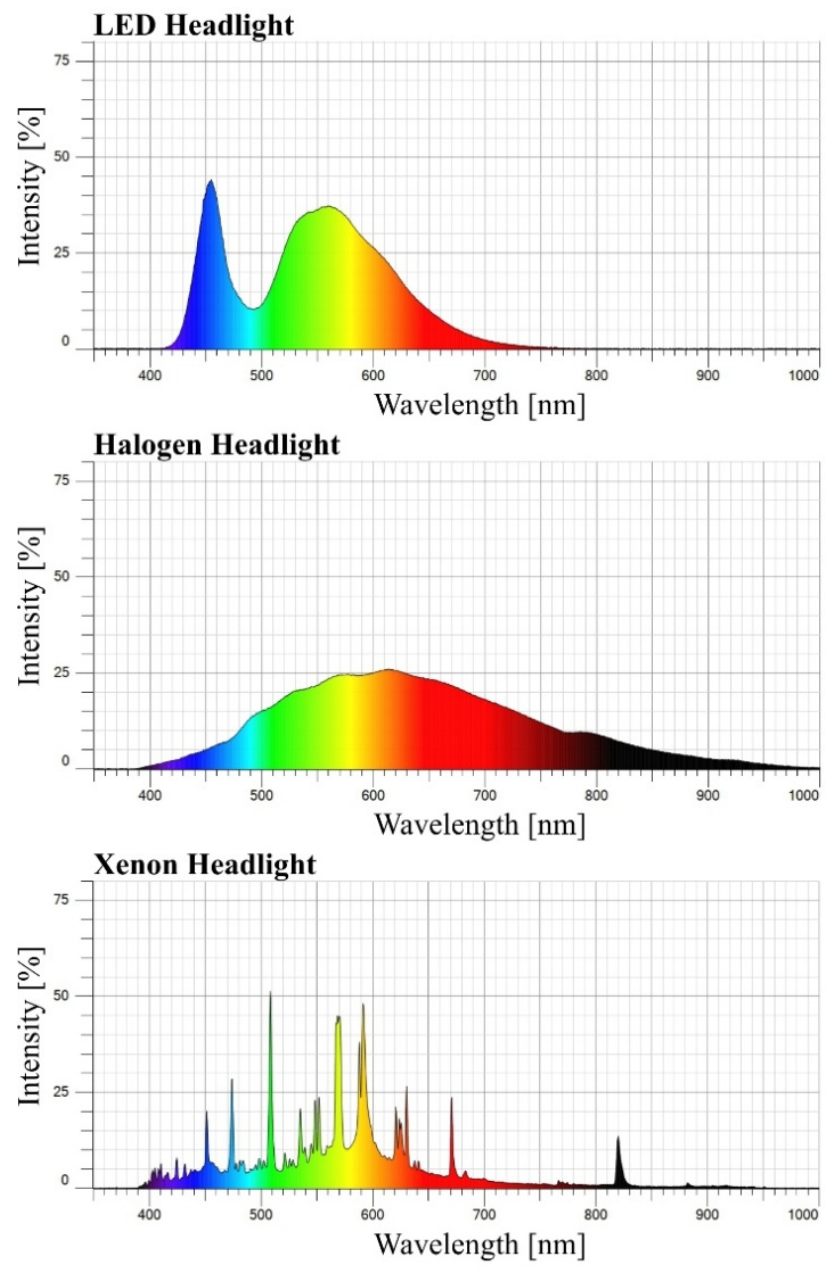

Figure 4. Recorded emission spectra of LED, halogen and xenon headlights

With the invention of an efficient blue-light LED it was possible to generate white light, combining a blue LED with special phosphors. The application of such white light LEDs in headlights is plausible because of their long lifetime and their compact design. Furthermore, the LEDs have a high luminosity and a small wattage [9]. They are often used as daytime running lights in cars but also as dimmed headlights in newer models. The emission spectrum of a LED headlight shows a typical peak at 450 nm (cf. Figure 4) belonging to the blue LED and the yellow color wavelengths belonging to the phosphor [10]. Today there are attempts to invent new phosphor mixtures 
to create an LED with a broad spectrum comparable to the emission spectrum of the sun.

In contrast to the white light of the LED, which has a high content of blue color wavelengths, the emission spectrum of the halogen headlight resembles the one of the sun (cf., Figure 4). Halogen lamps are continuum light sources and due to blackbody radiation and Planck's law they show their characteristically broad emission spectrum at their operating temperature (2500-3500 K) [11]. Most of today's cars still use the old halogen technique because of the relatively simple and reliable setup. However, this technique is slowly but steadily replaced by headlights with a higher luminosity such as xenon lamps [9].

A xenon light source is a gas discharge lamp filled with xenon gas. The lamp contains two electrodes ionizing the noble gas by a high voltage pulse. The conductive sphere enables a continuous flow of electricity, which leads to a very intense arc of light. Xenon lamps are line light sources as shown by the emission spectrum. The emitted wavelengths result in a white light similar to daylight. Such headlights have a wide lighting range but are expensive and have a complex setup because of the voltage regulation in the different stages (ignition, warmup, continuous operating) $[9,11]$.

A recent development in vehicular lighting systems is the laser light technology, available for instance in some BMW i8 models. A misunderstanding is the widespread assumption, that the system projects laser light directly onto the road. Instead, a high-power semiconductor laser diode emits blue light to a phosphor assembly, converting the light into white light [12]. The spectrum presented in Figure 5 has been taken from a BMW 7 Series car with combined high beam and daytime running light. To simulate a speed of $70 \mathrm{~km} / \mathrm{h}$ (minimum speed to switch on the laser light) the car was set on a test bench.

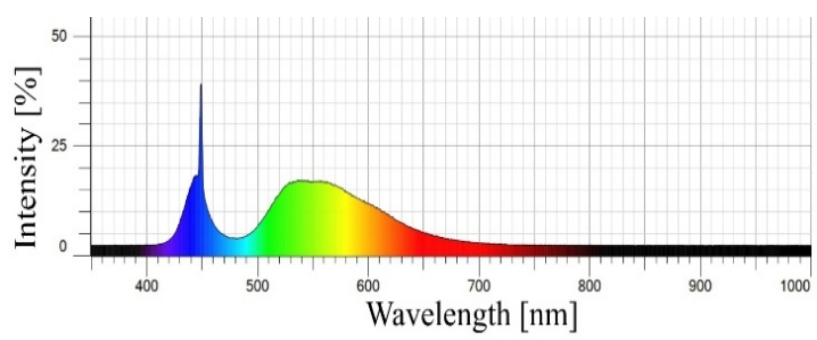

Figure 5. Emission spectrum of a laser headlight of a BMW 7 Series car

As can be seen in Figure 5, the resulting broadband spectrum consists of a remaining blue component and an energy down-converted spectrum with wavelengths between green and red perceived together as "white” light output.

\section{Typical Applications for Chemical Education}

\subsection{Qualitative Detection of Cations by Flame Test and Spectroscopy}

A well-known experiment in inorganic and analytical laboratory courses is the flame test to detect cations in a sample of unknown salts. The results of this experiment are often difficult to obtain because the color of the flame usually has to be determined simply with the eyes or through cobalt blue glasses. Moreover, sodium ions are universal impurities. Their flame color is in many cases so intense that it masks the color produced by other ions. If a sample contains more than one of these ions it is nearly impossible to detect the cations correctly without using a spectrometer. By using a device as described in chapter 3.2., the exact wavelengths of the emitted light can easily be determined, so that the cations can be detected precisely. In Figure 6, flame tests and emission spectra with lithium chloride and sodium chloride are shown as examples. The observed values are in very good agreement with the wavelengths given in [13].

Further emission spectra and photographs of flame tests of common cations can be downloaded from [14].
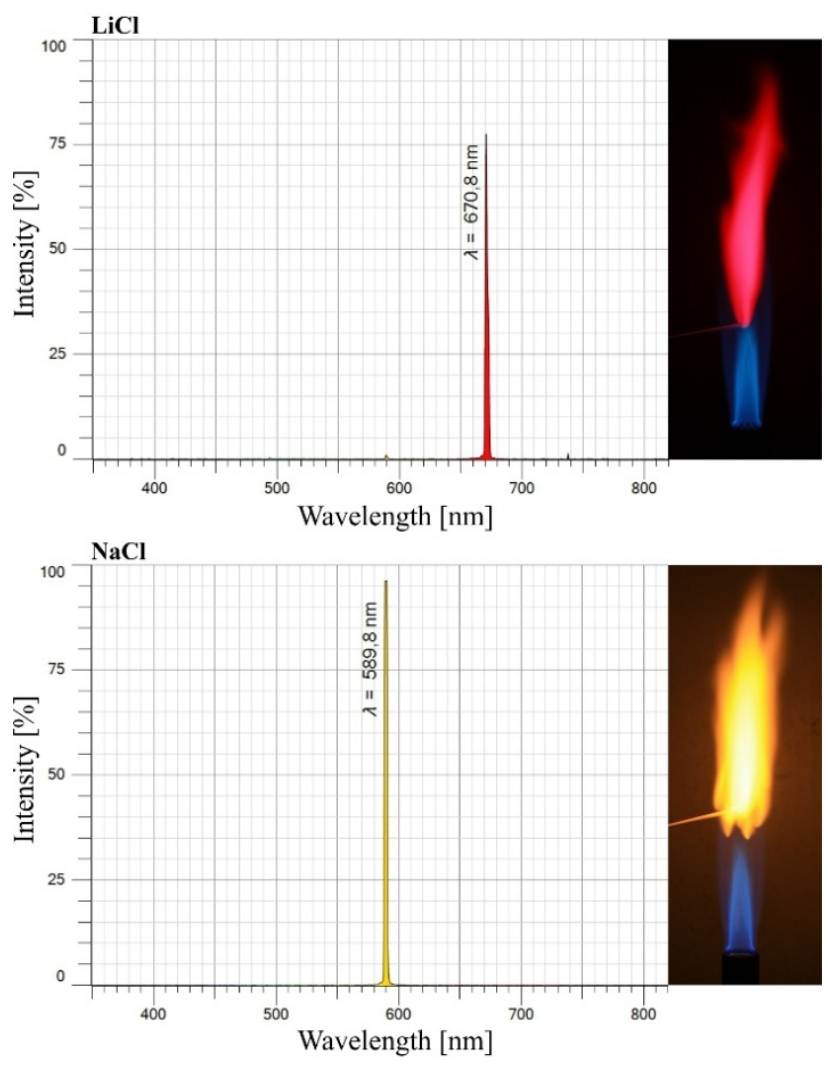

Figure 6. Emission spectra and photographs from the flame tests of lithium chloride $(\mathrm{LiCl})$ and sodium chloride $(\mathrm{NaCl})$

\subsection{Quantitative Analysis of Carmine}

One of the most important applications of UV/Vis spectroscopy are quantitative analyses, e.g. in food control. Consequently, in a final step an additional context-based experiment was developed to answer the following question: "How many insects are necessary to produce a packet of commercially available sherbet powder candies?"

The question gets an unexpected twist because the food additive carmine is obtained from insects.

\subsubsection{Background Information}

The coloration of food has a long tradition. To improve the goods for the trade and the consumer, toxic inorganic salts like lead chromate were added into groceries. These salts were replaced by synthetic organic dyes in the $19^{\text {th }}$ century. The production of natural dyes like carmine has 
decreased because synthetic dyes were cheaper and could be produced in a higher quality. However, since the synthetic Red No. 2 (amaranth) dye was banned in the US because it is suspected to be hazardous to health, the production of carmine is again on the rise [15].<smiles>Cc1c(C(=O)O)c(O)cc2c1C(=O)c1c(O)c(C3OC(CO)[C@H](O)[C@H](O)[C@H]3O)c(O)c(O)c1C2=O</smiles>

Figure 7. Structure of carminic acid

Carminic acid (3,5,6,8-tetrahydroxy-1-methyl-9,10dioxo-7-[3,4,5-trihydroxy-6-(hydroxymethyl)oxan-2-

yl]anthracene-2-carboxylic acid), as shown in Figure 7, is extracted from the female cochineal insect (Dactylopius coccus). These insects are bred on a certain prickly pear cactus and require a warm and dry climate. Therefore, the only efficient breeding places are in Algeria and on the Canary Islands. The female animals are about 100 days old when they are harvested by hand. When animals have reached an average weight of $46.96 \mathrm{mg}$ their content of the red dye is maximal [16]. Figure 8 shows some fullygrown insects on a cactus.

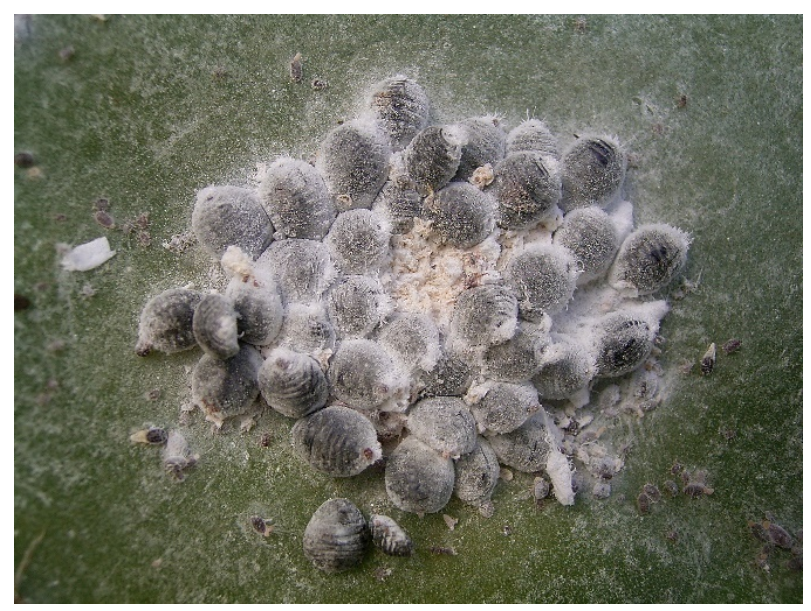

Figure 8. Fully-grown female cochineal insects just before harvest (photograph taken from Frank Vincentz, CC)

After the insects have been dried and ground to a black grain, the carminic acid is extracted with an acid or alkaline medium. Since one dried insect has an average weight of $10.74 \mathrm{mg}$, of which about $10 \%$ is carminic acid [16].

The carmine that is used in the food industry is a calcium and aluminum salt of the carminic acid. It is approved as food additive E120 in the European Union [17]. The hue of the red dye changes according to its $\mathrm{pH}$, being yellow at $\mathrm{pH} 4.8$ and red to purple above $\mathrm{pH} 6.2$ [16]. The undissolved salt of the carminic acid appears in a crimson red, as shown in Figure 9.

\subsubsection{Experimental Procedure and Results}

\subsubsection{Method}

To find out how many insects are necessary to produce a packet of $100 \mathrm{~g}$ of the default sherbet powder candies, the first step is to analyze the concentration of carmine in a weighed portion of candies. For a quantitative spectrometric analysis, one usually uses the LambertBeer-Bouguer Law,

$$
A=\varepsilon \cdot c \cdot d
$$

which relates the absorbance, A, to the concentration, c. In Eq. 2, $\varepsilon$ stands for the attenuation coefficient (at a given wavelength) and $d$ for the path length of radiation through the sample.

Using Eq. 2 for quantitative analysis (cf., e.g. $[18,19,20])$, a calibration curve has to be constructed. The observed absorbance at the wavelength $\lambda_{\max }$ is plotted against the analyte's concentration in a series of standards, where $\lambda_{\max }$ is the wavelength of the absorbance maximum of the analyte. The observed absorbance must be corrected by subtracting the absorbance of the blank (reference sample containing all the reagents except the analyte). As the next step, a linear calibration function can be generated from the calibration curve by least squares fit. The concentration of the series of standards should be in the same range as the estimated concentration of the analyte to get a significant calibration function [21]. The Lambert-Beer-Bouguer Law is only valid for ideal solutions in which the concentration of the analyte should not be higher than $10^{-3} \mathrm{~mol} / \mathrm{l}$ otherwise the variations are too wide [22].

\subsubsection{Apparatus and Reagents}

The absorbance measurements were made on an OceanOptics spectrometer with an additional cuvette holder, which has an integrated LED-boosted tungsten light source. The data were recorded and analyzed by using the software SpectraLab (LD Didactic, Germany).

The carmine was obtained from Waldeck (Germany) and a $0.1 \mathrm{M}$ sodium hydroxide solution (Carl Roth, Germany) was used as solvent. The analyzed sherbet powder candies, as shown in Figure 9, were products from Frigeo (Germany).

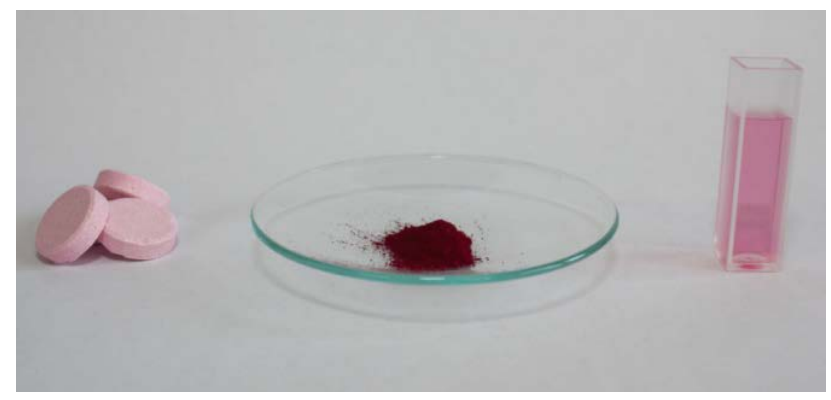

Figure 9. Analyzed sherbet powder candies (left), salt of the carminic acid undissolved (middle) and dissolved in sodium hydroxide solution (right)

\subsubsection{Procedure}

The candies were ground to a powder and $9.08 \mathrm{~g}$ of this powder were dissolved in sodium hydroxide solution. That solution was filtered and the filtrate was added to a $100 \mathrm{ml}$ graduated flask and filled up with the sodium hydroxide solution (standard sample $s_{1}$ ).

For the calibration line, $6.25 \mathrm{mg}$ of carmine was dissolved in $100 \mathrm{ml}$ sodium hydroxide solution. The 
dilution series was made using this solution (samples $s_{2}$ $\left.S_{5}\right)$.

The absorbance maximum $\lambda_{\max }$ of carmine in the sodium hydroxide solution was determined and the calibration function was calculated with the absorbance at $\lambda_{\max }$ of the dilution series.

The absorbance at $\lambda_{\text {max }}$ of the analysis sample was measured. With the help of the calibration function it is possible to determine the concentration of carmine in the sherbet powder candies.

\subsubsection{Results}

The absorbance maximum $\lambda_{\max }$ of the standard sample $s_{1}$ was obtained at $567 \mathrm{~nm}$, as shown in Figure 10.

Due to the weighed portion of carmine, the standard sample $s_{1}$ had a concentration of

$$
\begin{aligned}
\mathrm{c} & =\frac{\mathrm{m}_{\text {Carmine }}}{\mathrm{V}_{\mathrm{NaOH}} \cdot \mathrm{M}_{\text {Carmine }}} \\
& =\frac{6.25 \cdot 10^{-3} \mathrm{~g}}{100 \cdot 10^{-3} 1 \cdot 492.39 \frac{\mathrm{g}}{\mathrm{mol}}} \\
& =1.27 \cdot 10^{-4} \frac{\mathrm{mol}}{\mathrm{l}}
\end{aligned}
$$

The absorbance at $\lambda=567 \mathrm{~nm}$ of the dilution series was determined. The values are given in Table 1 together with the concentrations of the samples.

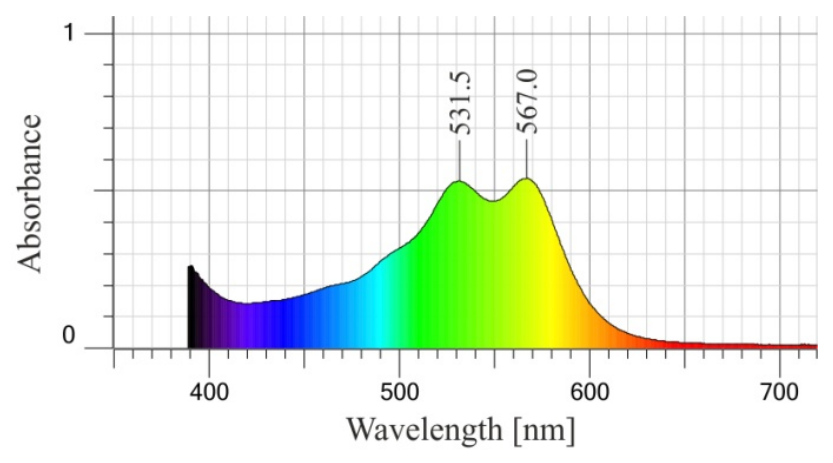

Figure 10. Absorption spectrum of carmine in sodium hydroxide solution (sample $s_{1}$ )

Table 1. Concentration and absorbance of the standard sample $s_{1}$ and the dilution series $s_{2}-s_{5}$ at $\lambda=567 \mathrm{~nm}$

\begin{tabular}{|c|c|c|}
\hline Sample & Concentration $[\mathrm{mol} / \mathrm{l}]$ & Absorbance \\
\hline 1 & $1.27 \cdot 10^{-4}$ & 0.499 \\
\hline 2 & $9.53 \cdot 10^{-5}$ & 0.418 \\
\hline 3 & $6.35 \cdot 10^{-5}$ & 0.255 \\
\hline 4 & $3.18 \cdot 10^{-5}$ & 0.120 \\
\hline 5 & $1.27 \cdot 10^{-5}$ & 0.037 \\
\hline
\end{tabular}

A five-point calibration was made. The resulting linear regression function - shown in Figure 11 - is set as calibration function with a coefficient of determination of 0.99631. The mathematical expression of the calibration function is:

$$
\mathrm{A}=4067 \frac{\mathrm{l}}{\mathrm{mol}} \cdot \mathrm{C}
$$

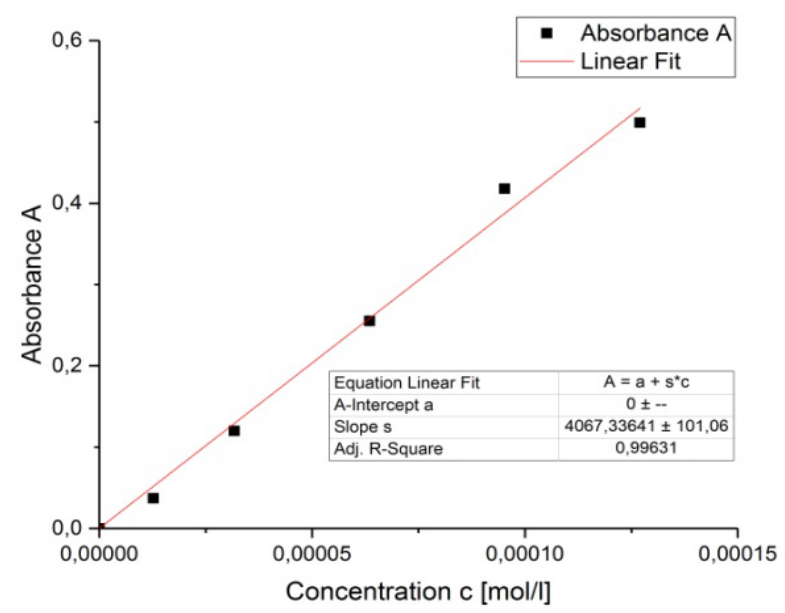

Figure 11. Five-point calibration with linear regression line

The absorbance of the analysis sample at $567 \mathrm{~nm}$ was $A_{\text {ana }}=0.260$. With Eq. 4 the concentration of carmine in the sample could be calculated as

$$
\mathbf{c}_{\text {carmine }}=\frac{0.260}{4067 \frac{\mathrm{l}}{\mathbf{m o l}}}=6.39 \cdot 10^{-5} \frac{\mathbf{~ m o l}}{\mathbf{l}}
$$

The concentration of carmine in $9.08 \mathrm{~g}$ of the candies conforms to a mass of $\mathrm{m}_{\text {carmine }}=3.15 \mathrm{mg}$ with Eq. 3 .

Thus, to produce a $100 \mathrm{~g}$ packet of the sherbet powder candies, $35 \mathrm{mg}$ of the carmine dye is necessary.

On the assumption that one dried cochineal insect contains about $10 \%$ carminic acid [16], about 33 insects are needed to produce one packet of sherbet powder candies.

\section{Conclusion}

In this contribution, a phenomenological and experimental access to basic principles of the interaction of electromagnetic radiation with matter was given using the example of $\mathrm{UV} / \mathrm{Vis}$ spectroscopy. In contrast to the traditional, theory-loaded way of teaching, the introduction described starts with an explanation of the functions of a spectrometer and its main parts. Therefore, a self-made, low-cost spectrometer with movable parts was constructed. Learners should explore the individual parts and their purpose in a spectrometer in respective experiments. After having been able to assemble such a spectrometer, learners should explore the instrument in practical applications. A commercial spectrometer can be used later for further studies since by then learners will have acquired enough knowledge about the general functionality of such devices.

In a final step, typical applications of UV/Vis spectroscopy like the qualitative detection of metal ions and quantitative analysis were implemented. The quantitative analysis experiment had an open question and a context-based trigger for motivation. This way, it was possible to link chemical applications with the everyday life. The more sophisticated theoretical part about the quantization of energy levels of matter can be taught following the 
described introduction. The learners can embed the fundamental theory of the interaction of radiation with matter into their pre-existing practical experience. This technique appears to provide easier access overall to spectroscopy in chemical education.

\section{Acknowledgements}

The authors thankfully acknowledge the financial support of this work by the DAAD (project number: 57236616). We thank Wolfgang Obert for technical support.

\section{References}

[1] Huntemann, H., Paschmann, A., Parchmann, I. and Ralle, B., "Chemie im Kontext - ein neues Konzept für den Chemieunterricht?,” CHEMKON, 6 (4), 191-196. Jan.1999.

[2] Ramsden, J., "How does a context-based approach influence understanding of key chemical ideas at 16+?,” International Journal of Science, 19 (6), 697-710. Jul.1997.

[3] Jones, M.B., Miller, C.R., "Chemistry in the Real World,” Journal of Chemical Education, 78 (4), 484-487. Apr.2001.

[4] Harel, I., Papert, S., Constructionism: research reports and essays, 1985-1990, Ablex Publishing Corporation, Norwood, 1991.

[5] Grasse, E.K., Torcasio M.H., Smith, A.W., "Teaching UV-Vis Spectroscopy with a 3D-Printable Smartphone Spectrophotometer," Journal of Chemical Education, 93 (1), 146-151. Jan.2016.

[6] Bougot-Robin, K., Atkins, S.C., Edel, J.B., "Optimization and Design of an Absorbance Spectrometer Controlled Using a Raspberry Pi To Improve Analytical Skills,” Journal of Chemical Education, 93 (7), 1232-1240. Jul.2016.

[7] Streidel, K., Introduction to Spectroscopy, Würzburg, 2016. [EManual] Available:

http://www.didaktik.chemie.uni-

wuerzburg.de/lehrer/materialien_fuer_den_einsatz_in_entwicklun gslaendern/spektroskopie/.
[8] Skoog, D.A., Holler, F.J., Stanley, R.C., Instrumentelle Analytik, Springer Spektrum, Berlin, 2013.

[9] Khanh, T.Q., Huhn, W., "Sichtverbesserungssysteme, “ Handbuch Fahrerassistenzsysteme, Springer Vieweg, Wiesbaden, 2015, 815-839.

[10] Kon, T., Kusano, T., "White LED with Excellent Rendering of Daylight Spectrum,” Optik \& Photonik, 9 (4), 62-65. Dec.2014.

[11] Schmidt, W., Optical spectroscopy in chemistry and life sciences, Wiley-VCH, Weinheim, 2005.

[12] Ulrich, L., "Whiter brights with lasers,” IEEE Spectrum, 50 (11), 36-56. Nov.2013.

[13] Jander, G., Blasius, E., Lehrbuch der analytischen und präparativen anorganischen Chemie, S. Hirzel Verlag, Stuttgart, 2006.

[14] Schairer, P., Kationennachweise mittels Flammenfärbung, Würzburg, 2017. [Online] Available: http://www.didaktik.chemie.uni-wuerzburg.de/lehrer/experimentezur-spektroskopie/.

[15] Sharma, V., McKone, H.T., Markow, P.G., “A Global Perspective on the History, Use, and Identification of Synthetic Food Dyes," Journal of Chemical Education, (88) 1, 24-28. Jan.2011.

[16] Baranyovits, F.L.C., "Cochineal carmine: an ancient dye with a modern role,” Endeavour, 2 (2), 85-92. Jan.1978.

[17] Lueck, E., Glandorf, K.K., Lexikon Lebensmittelzusatzstoffe, Behr, Hamburg, 1992.

[18] Karge, H.G., Geidel, E., "Vibrational Spectroscopy,” Molecular Sieves - Science and Technology, Springer Verlag, Berlin, 2004, 1-200.

[19] Harris, D.C., Exploring Chemical Analysis, W.H. Freeman and Company, New York, 2001.

[20] Checchetti, A., „Quantitative Analysis of Alcoholic Drinks. Use of Calibration Curve Method to Determine the Alcoholic Degree of Samples of Paesanella, a Distillate of the Family of Grappa," World Journal of Chemical Education, 3 (3), 70-73. May2015.

[21] Matter, L., Lebensmittel- und Umweltanalytik mit der Spektrometrie, VCH, Weinheim, 1995.

[22] Otto, M., Analytische Chemie, Wiley-VCH, Weinheim, 2011. 